\title{
UTILIDAD DE LA DETERMINACIÓN DE LA PRESIÓN ABDOMINAL DE FUGA EN EL DIAGNÓSTICO DE LA INCONTINENCIA DE ORINA FEMENINA EN LA ÉPOCA DEL TVT
}

\author{
J.L. GUTIÉRREZ BAÑOS, B. MARTÍN GARCÍA, J.A. PORTILLO MARTÍN, \\ J.I. DEL VALLE SCHAAN, R. HERNÁNDEZ RODRÍGUEZ, M.Á. CORREAS GÓMEZ, \\ A. ROCA EDREIRA, F. RUIZ IZQUIERDO, C. AGUILERA TUBET
}

\begin{abstract}
Servicio de Urología. Hospital Universitario Valdecilla. Santander (Cantabria).
\end{abstract}
Actas Urol Esp. 28 (7): 506-512, 2004

\section{RESUMEN}

UTILIDAD DE LA DETERMINACIÓN DE LA PRESIÓN ABDOMINAL DE FUGA EN EL DIAGNÓSTICO DE LA INCONTINENCIA DE ORINA FEMENINA EN LA ÉPOCA DEL TVT

OBJETIVO: Analizar los resultados del tratamiento de la incontinencia de esfuerzo tratada con TVT en función de la presión abdominal de fuga (PAF).

MATERIAL Y MÉTODO: Estudio retrospectivo de 52 pacientes operadas entre 1999 y 2002 que tenían estudio urodinámico y determinación de la PAF, con seguimiento mínimo de 3 meses. Se analizan las características clínicas y resultados respecto de la desaparición de la incontinencia al esfuerzo como de la sensación subjetiva y satisfacción de las pacientes. Denominamos grupo 1 si la PAF>100; grupo 2 entre 61 y 100; y grupo 3 si la PAF $<60$.

RESULTADOS: En el grupo 1 había 19 pacientes, 17 en el 2 y 16 en el 3 . No existieron diferencias entre los 3 grupos respecto a la edad, paridad, menopausia, histerectomía, años de evolución, cirugía previa, presencia y grado de cistocele, asociación de colporrafia al TVT y tipo de anestesia utilizada. El grado clínico de Obrink aumentó a medida que disminuía la PAF (grado 3 en 26,32\% del grupo 1; 31,58\% del 2 y $68,71 \%$ del 3 ).

La continencia al esfuerzo se consiguió en el $100 \%$ de las pacientes de los grupos 1 y 2 , y en el 93,75\% del grupo 3. La aparición de complicaciones, especialmente inestabilidad de novo o cuadros de urgencia-frecuencia así como la persistencia de inestabilidad en algunos casos de incontinencia mixta, hizo que el grado de satisfacción de las pacientes fuese del $79 \%$ en el grupo $1,76,5 \%$ en el $2 \mathrm{y}$ del $62,5 \%$ en el 3.

CONCLUSIONES: La determinación de la PAF no parece influir en la decisión de implantar un TVT, pero sí permite diferenciar un grupo $(\mathrm{PAF}<60 \mathrm{~cm}$ de agua) cuyos resultados esperados son ligeramente peores.

PALABRAS CLAVE: Incontinencia urinaria de esfuerzo. TVT. Presión abdominal de fuga. Uretropexia.

\section{ABSTRACT}

USEFULNESS OF ABDOMINAL LEAK POINT PRESSURE DETERMINATION IN THE DIAGNOSIS OF FEMALE URINARY INCONTINENCE IN THE TVT ERA

OBJECTIVE: To analyse our results about continence in the treatment of female urinary incontinence with the tension-free vaginal tape (TVT) procedure based on abdominal leak point pressure (ALPP).

PATIENTS AND METHODS: Retrospective study of the fifty two patients who had urodynamic study and abdominal leak point pressure determination and were operated on between 1999 and 2002 for stress urinary incontinence. We reviewed the data of clinical history, physical examination and urodynamic report, surgery, complications, and objective and subjective results. Those patients having ALPP>100 are included in group 1, between 61 and 100 in group 2 and less than 60 in group 3.

RESULTS: There were 19 patients in group 1, 17 in group 2 and 16 in group 3. We found no difference between the three groups regarding age, delivery, menopause, hysterectomy, evolution, previous surgery, grade of cystocele, association of anterior colporraphy to TVT and type of anaesthesia. The Obrink clinical grade increased as the ALPP decreased (grade 3 in $26.32 \%$ of group 1, 31.58\% in group 2 and $68.71 \%$ in group 3).

$100 \%$ of patients in groups 1 and 2 were continent with effort and $93.75 \%$ in group 3. Complications, especially de novo instability or urgency-frequency episodes or persistence of instability in patients having mixed incontinence, caused a decrease in the satisfaction degree to $79 \%$ in group $1,76.5 \%$ in 2 and $62.5 \%$ in 3 .

CONCLUSIONS: Abdominal leak point pressure determination does not change our decision of perform a TVT procedure but permits us to differentiate one group in which results could be worse.

KEY WORDS: Stress urinary incontinence. TVT. Abdominal leak point pressure. Uretropexy 
$\mathrm{L}$ a incontinencia urinaria de stress afecta al $20 \%$ de las mujeres por encima de los 45 años provocando un elevado coste social y económico ${ }^{1}$. Se han descrito multitud de técnicas para corregirla, la mayoría destinadas a elevar el cuello vesical y reforzar el tejido vaginal suburetral pero, a excepción de las de cabestrillo, no dan un adecuado soporte a la uretra ${ }^{2,3}$. Basado en la hipótesis de "la hamaca" de DeLancey", según la cual los incrementos en la presión uretral de cierre en las maniobras de stress se deben a que la uretra es comprimida contra un soporte a modo de hamaca, Ulmsten en $1996^{5}$ desarrolló la técnica de la cinta libre de tensión (TVT) colocada en la uretra media recreando el soporte suburetral con una malla de prolene. Desde su introducción su uso se ha disparado cambiando drásticamente el tratamiento de la incontinencia de esfuerzo en la mujer al ser un procedimiento mínimamente invasivo, de fácil aprendizaje y buenos resultados $(85-95 \%)^{6}$.

El concepto de presión abdominal de fuga (PAF) fue introducido por McGuire ${ }^{7}$ y se refiere a la presión intravesical en el momento de la pérdida de orina producida por maniobras que aumentan la presión abdominal en ausencia de contracción del detrusor ${ }^{8,9}$. Permite distinguir 3 tipos de incontinencia urinaria de esfuerzo: hipermotilidad uretral cuando la PAF es superior a $100 \mathrm{~cm}$ de agua, insuficiencia intrínseca del esfínter cuando la PAF es inferior a $60 \mathrm{~cm} \mathrm{de}$ agua y un transtorno mixto en los casos de PAF entre 60 y $100 \mathrm{~cm}$ de agua; estas diferencias tienen implicaciones terapéuticas ${ }^{8,10-13}$.

Estudiamos si el tratamiento de la incontinencia de orina femenina mediante la colocación de cintas suburetrales libres de tensión ofrece el mismo resultado independientemente de la PAF.

\section{OBJ ETIVO}

Analizar los resultados de nuestra serie en el tratamiento de la incontinencia femenina mediante el implante suburetral de cintas libres de tensión (TVT) en función de la PAF.

Valorar si el conocimiento de dicho parámetro nos permite conocer si algún grupo de pacientes no es subsidiario de dicho tratamiento o conlleva un mayor riesgo de complicaciones o fracaso.

\section{MATERIAL Y MÉTODOS}

Estudio retrospectivo de las 52 pacientes con incontinencia urinaria operadas entre 1999 y 2002 mediante la técnica de cinta libre de tensión en uretra media (TVT) descrita por Ulmstem ${ }^{5}$, a las que se les había practicado estudio urodinámico.

Las pacientes fueron evaluadas mediante historia clínica y exploración física; el grado clínico de incontinencia utilizado fue el de IngelmanSundberg ${ }^{14}$ (G1 incontinencia a la tos y estornudo, G2 al correr y levantar peso, G3 al andar y subir escaleras); el grado de cistocele se clasificó de acuerdo a la terminología de la Sociedad Internacional de la Continencia ${ }^{15}$. El estudio urodinámico se realizó con el equipo de urodinámica Phoenix 5000 de Albyn Medical version V.2.12, realizándose flujometría, cistomanometría, test $\mathrm{P} / \mathrm{Q}$ en los casos de flujometría patológica, $\mathrm{y}$ determinación de la presión abdominal de fuga al Valsalva ${ }^{7}$.

Para determinar la presión abdominal de fuga se ordena a la paciente hacer Valsalva hasta observar escape de orina, inicialmente con 200 cc en vejiga y si no se produce se sigue el llenado hasta 300 y $400 \mathrm{cc}$; si no se produce escape con Valsalva se la ordena toser. Consideramos como presión abdominal de fuga la presión intravesical que ocurre durante maniobras de incremento de la presión abdominal y en ausencia de contracción del detrusor que provoca escape de orina. En función de la misma formamos 3 grupos: grupo 1 con PAF superior a $100 \mathrm{~cm}$ de agua, grupo 2 entre 60 y 100 y grupo 3 con PAF inferior a $60 \mathrm{~cm}$ de agua.

La técnica quirúrgica fue la descrita por Ulmstem $^{5}$, utilizando anestesia preferentemente raquídea o general a la local. En las pacientes con cistocele grado 3 se propuso colporrafia anterior asociada al TVT.

Evaluamos los resultados respecto de la continencia, satisfacción de las pacientes y complicaciones. Se realizó estudio estadístico con el programa SPSS; para las variables cualitativas se utilizó el test de CHI-cuadrado y el test exacto de Fisher cuando fue necesario; para las variables cuantitativas se utilizó el análisis de varianza, se usó el test de Levene o test de homogeneidad de varianzas o pre-test de la ANOVA para ver si se 
podía utilizar el método paramétrico ANOVA y el test de Scheffe o post-test de la ANOVA para las comparaciones múltiples, en el caso de la variable retención post-operatoria se utilizó el test no paramétrico de la U de Mann-Withney debido al bajo tamaño muestral.

\section{RESULTADOS}

En total operamos 52 pacientes. El seguimiento oscila entre 3 y 48 meses (media 23,54 \pm 13,23 meses).

En el grupo 1 había 19 pacientes, en el 2 había 17 y en el grupo 3 había 16 pacientes.

En la Tabla 1 se observan los datos referentes a la edad, paridad, menopausia, histerectomía, años de evolución y presencia de urgencia en cada grupo, sin que se observen diferencias entre ellos, si bien las pacientes del grupo 3 tienden a ser más añosas.

El grado clínico de incontinencia se observa en la Tabla 2, se puede ver como aumenta el grado clínico a medida que disminuye la PAF siendo la diferencia significativa en el test de CHI-cuadrado $(p=0,015)$. Existe una cierta dispersión de valores pudiendo observarse casos de PAF de 60 en pacientes con grado clínico 1 o de 116 en pacientes con grado clínico 3 .

La exploración física demostró cistocele de diversos grados en 24 pacientes $(46,15 \%)$, no estando reflejado el dato en la historia clínica en 2 casos (Tabla 3), no se observaron diferencias entre los grupos ( $\mathrm{p}=0,388)$.

Se habían realizado 11 técnicas anti-incontinencia en 9 pacientes (Tabla 4); a una paciente se la había practicado un RAZ 1 y RAZ 3, 4 y 2 años respectivamente antes del implante de TVT y a otra se le había realizado un Kelly en 1983 y posteriormente se le implantaron microbalones de urovive; no existían diferencias entre los 3 grupos ( $p=0,534)$. La existencia de cirugía previa no dificultó la técnica quirúrgica; la comparación entre la duración de la intervención en pacientes previamente operadas $(\mathrm{X}=30 \pm 8,94$ minutos) y pacientes no operadas $(\mathrm{X}=30,85 \pm 8,61)$ no fue significativa $(\mathrm{p}=0,842)$.

Tabla 1. Características de las pacientes

\begin{tabular}{|c|c|c|c|c|c|c|c|}
\hline & $\mathbf{N}$ & Edad & Partos & Evolución & Menopausia & Histerectomía & Urgencia \\
\hline $\begin{array}{l}\text { Grupo } 1 \\
\text { PAF }>100\end{array}$ & 19 & $\begin{array}{c}55,47 \pm 8,17 \\
(43-69)\end{array}$ & $\begin{array}{c}2,71 \pm 1,40 \\
(1-6)\end{array}$ & $\begin{array}{l}7,21 \pm 4,93 \\
(3-20)\end{array}$ & $\begin{array}{l}\text { SI: } 15 \text { (79\%) } \\
\text { NO: } 4 \text { (21\%) }\end{array}$ & $\begin{array}{c}\text { SI: } 9(47,3 \%) \\
\text { NO: } 10(52,7 \%)\end{array}$ & $\begin{array}{l}\text { SI: } 5(26,3 \%) \\
\text { NO: } 14(73,7)\end{array}$ \\
\hline $\begin{array}{l}\text { Grupo } 2 \\
\text { PAF 60-100 }\end{array}$ & 17 & $\begin{array}{c}58,24 \pm 9,94 \\
(42-72)\end{array}$ & $\begin{array}{c}3 \pm 1,7 \\
(1-7)\end{array}$ & $\begin{array}{c}8,44 \pm 10,63 \\
(1-40)\end{array}$ & $\begin{array}{l}\text { SI: } 15(88,2 \%) \\
\text { NO: } 2(11,8 \%)\end{array}$ & $\begin{array}{c}\text { SI: } 6(35,3 \%) \\
\text { NO: } 11(64,7 \%)\end{array}$ & $\begin{array}{c}\text { SI: } 6(35,3 \%) \\
\text { NO: } 11(64,7 \%)\end{array}$ \\
\hline $\begin{array}{l}\text { Grupo } 3 \\
\text { PAF }<60\end{array}$ & 16 & $\begin{array}{c}61,94 \pm 10,43 \\
(40-75)\end{array}$ & $\begin{array}{c}3,47 \pm 1,81 \\
(0-7)\end{array}$ & $\begin{array}{l}8,67 \pm 8,27 \\
\quad(2-30)\end{array}$ & $\begin{array}{l}\text { SI: } 14(87,5 \%) \\
\text { NO: } 2(12,5 \%)\end{array}$ & $\begin{array}{c}\text { SI: } 5(31,25 \%) \\
\text { NO: } 11(68,75 \%)\end{array}$ & $\begin{array}{c}\text { SI: } 5(31,25 \%) \\
\text { NO: } 11(68,75 \%)\end{array}$ \\
\hline $\mathbf{p}$ & & 0,136 & 0,426 & 0,289 & 0,710 & 0,358 & 0,488 \\
\hline
\end{tabular}

Tabla 2. Grado clínico de incontinencia. PAF en cada grupo

\begin{tabular}{cccccc}
\hline $\begin{array}{c}\text { Grado } \\
\text { clínico }\end{array}$ & $\begin{array}{c}\text { Grupo 1 } \\
\text { PAF }>\mathbf{1 0 0}\end{array}$ & $\begin{array}{c}\text { Grupo 2 } \\
\text { PAF 60-100 }\end{array}$ & $\begin{array}{c}\text { Grupo 3 } \\
\text { PAF } \mathbf{6 0}\end{array}$ & Total & $\begin{array}{c}\text { PAF } \\
\text { (cm agua) }\end{array}$ \\
\hline 1 & $2(10,5 \%)$ & 0 & $1(6,25 \%)$ & $3(5,8 \%)$ & $\begin{array}{c}94,67 \pm 31,07 \\
(60-120)\end{array}$ \\
2 & $12(63,2 \%)$ & $13(68,42 \%)$ & $4(25 \%)$ & $29(55,8 \%)$ & $86,76 \pm 27,08$ \\
$(20-140)$ & & $20(38,4 \%)$ & $63,53 \pm 35,16$ \\
3 & $5(26,3 \%)$ & $4(31,58 \%)$ & $11(68,75 \%)$ & & $(20-116)$ \\
\hline
\end{tabular}

$\mathrm{P}=0,015$ 
Tabla 3. Cistocele por grupos

\begin{tabular}{lcccc}
\hline Cistocele & Grupo 1 & Grupo 2 & Grupo 3 & Total \\
\hline Ausente & $8(42,1 \%)$ & $10(58,8 \%)$ & $8(50 \%)$ & $26(50 \%)$ \\
Grado 1 & $5(26,3 \%)$ & $4(23,5 \%)$ & $1(6,25 \%)$ & $10(19,23 \%)$ \\
Grado 2 & $3(15,8 \%)$ & $3(17,7 \%)$ & $5(31,25 \%)$ & $11(21,15 \%)$ \\
Grado 3 & $2(10,6 \%)$ & 0 & $1(6,25 \%)$ & $3(5,77 \%)$ \\
Sin datos & $1(5,25 \%)$ & 0 & $1(6,25 \%)$ & $2(3,85 \%)$ \\
\hline P=0,388 & & & &
\end{tabular}

Tabla 4. Intervenciones quirúrgicas previas (11 en 9 pacientes)

\begin{tabular}{lccc}
\hline Intervención & Grupo 1 & Grupo 2 & Grupo 3 \\
\hline Kelly & 1 & & 1 \\
Raz 1 & 1 & 2 & \\
Stamey & & 1 & \\
Gittes & & 1 & \\
Raz 3 & 1 & & \\
Burch Laparos & 1 & & \\
Urovive & & $4(23,53 \%)$ & $3(18,75 \%)$ \\
Total & $4(21,05 \%)$ & & \\
\hline
\end{tabular}

$\mathrm{P}=0,534$

En 6 pacientes se realizaron procedimientos asociados sin que existieran diferencias entre los 3 grupos ( $\mathrm{p}=0,344)$; en un caso se extrajo endoscópicamente una grapa intravesical de un Burch laparoscópico previo y en los otros 5 se realizó colporrafia anterior, 3 por cistocele grado 3 y 2 por cistocele grado 2 (Tabla 5).

Se diagnosticaron 9 casos de incontinencia mixta (17,31\%); 3 en el grupo 1 (15,8\%), 4 en el 2 $(23,53 \%)$ y 2 en el $3(12,5 \%)$, diferencias no significativas $(p=0,734)$.

La intervención se realizó con anestesia local en 1 caso, en 29 con anestesia raquídea y en 22 general (Tabla 6), diferencias no significativas $(\mathrm{p}=0,741)$.

Como complicaciones cabe destacar la retención post-operatoria y la inestabilidad de novo (Tabla 7). Tuvimos 8 casos de retención post-operatoria $(15,38 \%)$ con una duración media de 14,28
Tabla 5. Procedimientos asociados

\begin{tabular}{lcccc}
\hline Procedimiento & Grupo 1 & Grupo 2 & Grupo 3 & Total \\
\hline Colporrafia & 3 & 1 & 1 & 5 \\
$\begin{array}{l}\text { Extracción } \\
\text { de grapa } \\
\text { intravesical }\end{array}$ & 1 & 0 & 0 & 1 \\
\hline
\end{tabular}

$\mathrm{P}=0,344$

Tabla 6. Tipo de anestesia

\begin{tabular}{lcccc}
\hline Anestesia & Grupo 1 & Grupo 2 & Grupo 3 & Total \\
\hline Local & 0 & $1(5,88 \%)$ & 0 & $1(1,92 \%)$ \\
General & $8(42,11 \%)$ & $6(35,3 \%)$ & $8(50 \%)$ & $22(42,31 \%)$ \\
Raquídea & $11(57,89 \%)$ & $10(58,82 \%)$ & $8(50 \%)$ & $29(55,77 \%)$ \\
\hline
\end{tabular}

$\mathrm{P}=0,741$

Tabla 7. Complicaciones

\begin{tabular}{lcccc}
\hline Complicación & Total & Grupo 1 & Grupo 2 & Grupo 3 \\
\hline Retención & $8(15,38 \%)$ & $5(26,32 \%)$ & $1(5,88 \%)$ & $2(12,5 \%)$ \\
$\begin{array}{l}\text { Inestabilidad } \\
\text { de novo }\end{array}$ & $7(13,46 \%)$ & $2(10,53 \%)$ & $2(11,76 \%)$ & $3(18,75 \%)$ \\
$\begin{array}{l}\text { Dolor perineal } \\
\text { Hematoma }\end{array}$ & $1(1,92 \%)$ & $1(5,26 \%)$ & & \\
$\begin{array}{l}\text { Perforación } \\
\text { vesical }\end{array}$ & $3(5,77 \%)$ & $2(10,53 \%)$ & $1(5,88 \%)$ & $1(6,25 \%)$ \\
\hline
\end{tabular}

Retención: $\mathrm{p}=0,213$

Inestabilidad de novo: $\mathrm{p}=0,882$

$\pm 18,52$ días (rango 2-60) sin que las diferencias fuesen significativas entre los grupos $(\mathrm{p}=0,213), 6$ de ellos se resolvieron en menos de 7 días y 7 en menos de 2 semanas. Inestabilidad de novo vimos en 7 pacientes $(13,46 \%), 2(10,5 \%)$ en grupo 1,2 $(11,7 \%)$ en grupo 2 y $3(18,7 \%)$ en el grupo 3 , diferencias no significativas $(\mathrm{p}=0,882)$; 3 eran pacientes previamente operadas y otra una paciente a la que se realizó colporrafia anterior. Solamente 2 pacientes han respondido de forma adecuada al tratamiento anticolinérgico. También se observó 1 hematoma pélvico que no precisó ninguna actuación y 1 caso de dolor perineal. 
Se logró continencia al stress en el $100 \%$ de las pacientes de los grupos 1 y 2 y en el 93,75\% del 3 ( 1 solo fracaso), diferencia no significativa $(\mathrm{p}=0,308)$. Sin embargo, sólo el $79 \%$ de las pacientes en el grupo $1,76,5 \%$ en el 2 y $62,5 \%$ en el 3 estaban satisfechas del resultado obtenido, diferencia no significativa $(p=0,998)$. La inestabilidad de novo y la persistencia de síntomas en pacientes con incontinencia mixta fueron las causas principales de insatisfacción (Tabla 8).

\section{COMENTARIOS}

La determinación de la PAF no está estandarizada y su papel en el estudio de la incontinencia de orina femenina no está claro ${ }^{16}$. Algunos autores le encuentran de escasa utilidad dado su baja reproducibilidad debido a que los resultados se pueden ver influenciados por factores tales como la presencia de catéter vesical, volumen vesical, la capacidad de las pacientes para generar presión con Valsalva y el importante solapamiento existente entre continentes e incontinentes ${ }^{17-20}$. Frente a éstos, otros autores refieren que es un test reproducible $^{21-23}$ e inclusive Finazzy y cols. $^{24}$ no encuentran variaciones en función de la presencia o no de catéter vesical y la posición de la paciente. Sí es necesario tener en cuenta la presencia de prolapso genital que puede generar falsas PAF elevadas y la capacidad de la paciente para generar presión, siendo aconsejable hacerlo con volúmenes vesicales entre 200 y $300 \mathrm{cc}^{10,25,26}$.

Las pacientes con PAF baja, inferior a $60 \mathrm{~cm}$ de agua, presentan un transtorno intrínseco del esfínter y responden mejor al tratamiento mediante técnicas de "bulking", sling o esfínter artificial; por el contrario, las pacientes con PAF superior a $100 \mathrm{~cm}$ de agua tienen como transtorno fundamental la hipermotilidad uretral y pueden ser tratadas con éxito mediante técnicas de suspensión; el grupo intermedio (PAF entre 60 y $100 \mathrm{~cm}$ de agua) es una mezcla de las otras dos y el tratamiento ideal serían los slings aunque también pueden responder a las suspensiones $^{8,10-13}$.

El hecho de que el procedimiento quirúrgico con TVT sea de fácil aprendizaje, mínimamente invasivo y con resultados satisfactorios ha hecho que su uso se halla generalizado ${ }^{6}$. Su mecanismo de acción reside en la corrección del soporte inadecuado de la uretra, debido a la alteración de los ligamentos pubouretrales y pared vaginal suburetral, creando una resistencia uretral dinámica al stress sin interferir la función uretral de reposo, ni elevar el cuello vesical ni reducir su movilidad $^{27-30}$; se observa un incremento en el índice de transmisión en la zona media de la uretra sin cambios en la presión uretral de cierre ${ }^{30,31}$.

No se han encontrado diferencias en los resultados en función de la edad, menopausia, obesidad, cirugías anti-incontinencia previas, tipo de anestesia y asociación a técnicas de reconstrucción del suelo pélvico ${ }^{32-45}$; Jacquetin ${ }^{46}$ refiere un mayor índice de retención post-operatoria en estas últimas pacientes.

Los resultados objetivos oscilan entre el 85 y 95\%33,36,37,47-52 mientras que los resultados subjetivos, al igual que en nuestra serie, son menores oscilando entre el $66 \%$ y $83 \%{ }^{31,37,51}$. La causa de esta disminución en el resultado subjetivo es debida a las complicaciones; las principales son la retención y la inestablidad de novo, independientes de la $\mathrm{PAF}^{37,51,53-57}$.

Tabla 8. Continencia y satisfacción

\begin{tabular}{lcccc}
\hline & Total & Grupo 1 & Grupo 2 & Grupo 3 \\
\hline Continencia al stress & $51(98 \%)$ & $19(100 \%)$ & $17(100 \%)$ & $15(93,75 \%)$ \\
Satisfacción & $39(75 \%)$ & $15(78,95 \%)$ & $13(76,47 \%)$ & $10(62,5 \%)$ \\
$\begin{array}{l}\text { Causas de } \\
\text { Insatisfacción }\end{array}$ & 1 urgencia mixta & $\begin{array}{c}2 \text { inestabilidades } \\
\text { urgencias mixtas } \\
1 \text { dolor perineal }\end{array}$ & $\begin{array}{l}2 \text { inestabilidades } \\
3 \text { inestabilidades }\end{array}$ & 1 fracaso \\
& & incontinencia mixta
\end{tabular}

Continencia: $\mathrm{p}=0,308$

Satisfacción: $p=0,998$ 
La influencia de la presión uretral de cierre o de la presión abdominal de fuga es discutible. En la serie de Deval y cols. ${ }^{37}$ no influyó en los resultados; Mutone y cols. ${ }^{31}$ refieren unos resultados objetivos y subjetivos del 91 y $83 \%$ respectivamente en pacientes con presión uretral de cierre inferior a $20 \mathrm{~cm}$ de agua y/o presión abdominal de fuga al Valsalva inferior a $60 \mathrm{~cm}$ de agua; mientras que para Jacquetin ${ }^{58}$ la presión uretral de cierre inferior a $30 \mathrm{~cm}$ de agua se asoció a una reducción de éxitos del 89 al $75 \%$ y Lo y cols. ${ }^{41}$ encuentran que los resultados descienden del $86,6 \%$ al $73 \%$ cuando existe una insuficiencia intrínseca del esfinter, resultados similares a los nuestros con resultados objetivos y subjetivos que descienden del 100 y $79 \%$ a 93,75 y $62,5 \%$, respectivamente, cuando la presión de fuga al Valsava es inferior a $60 \mathrm{~cm}$ de agua.

Aunque los resultados del tratamiento de la incontinencia de orina femenina en los casos de $\mathrm{PAF}$ inferior a $60 \mathrm{~cm}$ de agua son ligeramente inferiores, siguen siendo buenos (63 a 83\% de pacientes satisfechas) $)^{31,37,41,58}$, lo que asociado a su sencillez hace del implante de TVT un tratamiento efectivo en estas pacientes. El conocer la PAF nos parece innecesario para la elección del tratamiento, si bien nos permite conocer un grupo de pacientes con un mayor riesgo de fracaso.

\section{REFERENCIAS}

1. THOMAS TM, PLYMET KR, BLANNIN J, MEADE TW.: The prevalence of urinary stress incontinence. BMJ 1980; 281: 1243-1245.

2. LEACH GE, DMOCHOWSKI RR, APPELL RA et al.: Female stress urinary incontinence clinical guidelines panel summary report on surgical management of female stress urinary incontinence. J Urol 1997; 158: 875-880.

3. CARLIN B, KLUTKE J, KLUTKE C.: The tension-free vaginal tape procedure for the treatment of stress incontinence in the female patient. Urology 2000; 56 (Suppl 6): 28-31.

4. DELANCEY JOL.: Structural support of the urethra as it relates to stress urinary incontinence: the hammock hypothesis. Am J Obstet Gynecol 1994; 170: 1713-1723.

5. ULMSTEN U, HENRIKSSON L, JOHNSON P, VARHOS G.: An ambulatory surgical procedure under local anesthesia for treatment of female urinary incontinence. Int Urogynecol J Pelvic Floor Dysfunc 1996; 7: 81-86.

6. HAAB F, TRAXER O, CIOFU C.: Tension-free vaginal tape: why an unusual concept is so successful?. Current Opinion in Urology 2001; 11: 293-297.

7. MCGUIRE EJ, FITZPATRICK CC, WANG $J$ et al.: Clinical assessment of urethral sphincter function. J Urol 1993; 150: 1452-1454.

8. MCGUIRE EJ, O CONNELL HE.: Leak point pressures in stress incontinence; in Blaivas $\mathrm{J}$ and Chancellor M Atlas of urodynamics, edit by Williams and Wilkins, Baltimore (USA), 1996: 208-213.
9. HOMMA Y, BATISTA J, BAUER S et al.: Urodynamics; in Abrams $\mathrm{P}$, Cardozo L, Khoury $\mathrm{S}$ and Wein $\mathrm{A}, 2^{\text {nd }}$ International Consultation on Incontinence, edit by Plymbridge Distributers Ltd (United Kingdom): 317-372.

10. MCGUIRE EJ, CESPEDES RD, O CONNELL HE.: Leak point pressures. Urol Clin North Am 1996; 23: 253-262.

11. GHONIEM GM, ELGAMASY AN, ELSERGANY R, KAPOOR DS.: Grades of intrinsic sphincteric deficiency (ISD) associated with female stress urinary incontinence. Int Urogynecol J Pelvic Floor Dysfunct 2002; 13: 99-105.

12. MCGUIRE EJ.: Urodynamic evaluation of stress incontinence. Urol Clin North Am 1995; 22: 551-555.

13. MADJAR S, BALZARRO M, APPEL RA, TCHETGEN MB, NELSON D.: Baseline abdominal pressure and Valsalva leak point pressures-correlation with clinical and urodynamic data. Neurourol Urodyn 2003; 22: 2-6.

14. INGELMAN-SUNDBERG A, ULMSTEN U.: Surgical treatment of female urinary stress incontinence. Contr Gynecol Obstet 1983; 10: 51-54

15. ABRAMS P, BLAIVAS JG, STANTON SL et al.: The standarditation of terminology of low urinary tract function recommended by the International Continence Society. Int Urogynecol J Pelvic Floor Dysfunct 1990; 1: 45-58.

16. LOSE G.: Urethral pressure measuremnts; in Cardozo L and Staskin D, Textbook of female urology and urogynecology, edit by Isis Media, London (United Kingdom) 2001: 215-226.

17. SILTBERG H, LARSSON G, VICTOR A.: The reproducibility of a new method to measure leak point pressure in patients with GSI. Neurourol Urodyn 1994; 13: 456-458.

18. BUMP RC, ELSER DM, THEOFRASTOUS JP, MCCLISH DK.: Valsalva leak point pressures in women with genuine stress incontinence: reproducibility, effect of the catheter caliber and correlations with other measures of urethral resistance. Am J Obstet Gynecol 1995; 173: 551-557.

19. PALAO F, GALISTEO R, JIMÉNEZ A et al.: Relación entre la presión uretral de cierre y la presión abdominal de fuga en la incontinencia urinaria femenina de esfuerzo. Urod $A$ 2000; 13: 20-28.

20. HAAB F, CIOFU C, PADRON P et al.: Feasibility of Valsalva leak point pressure. Prospective study. Prog Urol 1997; 7: 611-614.

21. BUMP RC, ELSER DM, MCCLISH DK.: Valsalva leak point pressures in adult women with genuine stress incontinence: reproducibility effect of catheter caliber and correlations with passive urethral pressure profilometry. Neurourol Urodyn 1993; 12: 307-308.

22. NITTI VW, COMBS AJ.: Correlation of Valsalva leak point pressure with subjective degree of stress urinary incontinence in women. J Urol 1996; 155: 281-285.

23. PRIETO L, GARCÍA L, LLORENS F et al.: Presión mínima de escape con Valsalva: una útil aproximación a la incontinencia urinaria femenina tipo III. Arch Esp Urol 1998; 51: 783-789.

24. FINAZZI E, PARISI AI, PETTA F, D`AMICO A, VESPASIANI G.: Valsalva leak point pressure: can variables in its execution affect the results? Eur Urol Supplements 2003; 2: 117 (Abstract 457).

25. FAERBER GJ, VASLIN AR.: Variations in Valsalva leak point pressure with increasing vesical volume. $J$ Urol 1998; 159: 1909-1911.

26. MCGUIRE EJ, LENG WW.: Leak point pressures in Cardozo L and Staskin D, textbook of female urology and urogynecology, edit by Isis Media, London (United Kingdom) 2001: 227-237.

27. PETROS P, ULMSTEN U.: An integral theory and its method for the diagnosis and management of female urinary incontinence. Scand J Urol Nephrol 1993; 153: 1-93. 
28. HALASKA M, OTCENASEK M, MARTAN A, MASATA J, VOIGT R, SEIFERT M.: Pelvic anatomy changes after TVT procedure assessed by MRI. $24^{\text {th }}$ Annual Meeting of the International Urogynecoloy Association, august 23-26, 1999 Denver (USA). Int Urogynecol J Pelvic Floor Dysfunct 1999; 10 (Suppl 1): S88.

29. KLUTKE J, CARLIN B, KLUTKE C.: The tension-free vaginal tape procedure: correction of stress incontinence with miccional alteration in proximal urethral mobility. Urology 2000; 55: 512-514.

30. LO TS, WANG AC, HORNG SG, LIANG CC, SOONG YK.: Ultrasonographic and urodynamic evaluation after tension-free vaginal tape procedure (TVT). Acta Obstet Gynecol Scand 2001; 80: 65-70.

31. MUTONE N, MASTROPIETRO M, BRIZENDINE E, HALE D.: Effect of tension free vaginal tape procedure on urodynamic continence indices. Obstet Gynecol 2001; 98: 638-645.

32. LAVY Y, LEV-SAGIE A, HAMANI Y, HURWITS A, ADONI A.: Cough stress during the tension-free vaginal tape procedure: is it necessary? $32^{\text {nd }}$ Annual Meeting of the ICS august 28-30, 2002, Heildelberg (Alemania). Neurourol Urodynam 2002; 21: 4 (abstract 289).

33. LEBRET T, LUGAGNE PM, HERVÉ JM et al.: Evaluation of tension-free vaginal tape procedure. Eur Urol 2001; 40: 543-547.

34. ADAMIAK A, MILART P, SKORUPSKI P et al.: The efficacy and safety of the tension-free vaginal tape procedure do nor depend on the method of analgesia. Eur Urol 2002; 42: $29-33$.

35. LO TS, HUANG HJ, CHANG CL, WONG SY, HORNG SG, LIANG CC.: Use of intravenous anesthesia for tension-free vaginal tape therapy in elderly women with genuine stress incontinence. Urology 2002; 59: 349-353.

36. HAAB F, SANANES S, AMARENCO G et al.: Results of the tension-free vaginal tape procedure for the treatment of type II stress urinary incontinence at a minimum follow-up of 1 year. J Urol 2001; 165: 159-162.

37. DEVAL B, JEFFRY L, AL NAJJAR F, SORIANO D, DARAI E.: Determinants of patient dissatisfaction after a tension-free vaginal tape procedure for urinary incontinence. J Urol 2002; 167: 2093-2097.

38. MUKHERJEE K, CONSTANTINE G.: Urinary stress incontinence in obese women: tension-free vaginal tape is the answer. BJU International 2001; 88: 881-883.

39. AZAM U, FRAZER MI, KOZMAN EL, WARD K, HILTON P, RANE A.: The tension-free vaginal tape procedure in women with previous failed stress incontinence surgery. $J$ Urol 2001; 166: 554-556.

40. REZAPOUR M, ULMSTEN U.: Tension-free vaginal tape (TVT) in women with recurrent stress urinary incontinence. A long-term follow-up. Int Urogynecol J Pelvic Floor Dysfunct 2001; 12: 9-11.

41. LO TS, HORGN SG, CHANG CL, HUANG HJ, TSENG LH, LIANG CC.: Tension-free vaginal tape procedure after previous failure in incontinence surgery. Urology 2002; 60: 57-61.

42. GORDON D, GOLD R, PAUZNER D, LESSING J, GROUTZ A.: Combined genitourinary prolapse repair and prophylactic tension-free vaginal tape in women with severe prolapse and occult stress urinary incontinence: preliminary results. Urology 2001; 58: 547-550.

43. TAHORA H, MIYAJIMA S, OKADOME A, NABESHIMA Y, ARIYOSHI A.: The genitourinary prolapse repair combined with TVT: clinical outcomes and long-term follow up. $32^{\text {nd }}$ ICS Meeting, august 28-30, 2002, Heildelberg (Alemania). Neurourol Urodynam 2002; 21: 4 (abstract 531).
44. JOMAA M.: Combined tension-free vaginal tape and prolapse repair under local anesthesia in patients with symptoms of both urinary incontinence and prolapse. Gynecol Obstet Invest 2001; 51: 184-186.

45. MESCHIA M, PIFAROTTI P, GATTEI U, RONCHETTI A, STOPPELLI S, LAMPUGNANI F.: TVT and prolapse repair for treatment of occult stress urinary incontinence: a randomized study. $32^{\text {nd }}$ ICS Meeting, august 28-30, 2002, Heildelberg (Alemania). Neurourol Urodynam 2002; 21: 4 (abstract 299).

46. JACQUETIN B, FATTON B, FERNÁNDEZ M.: Comparative results of isolated TVT versus prolapse cure associated TVT: 171 cases with one to three years follow up. $31^{\text {st }}$ ICS Annual Meeting, September 2-5, 2000, Tampere (Finlandia). Neurourol Urodynam 2000; 19: 4, (abstract 219).

47. CARLIN BI, KLUTKE JJ, KLUTLE CG.: The tension-free vaginal tape procedure for the treatment of stress incontinence in the female patient. Urology 2000; 56 (Suppl 6A): 28-31.

48. MORAN PA, WARD KL, JOHNSON D, SMIRNI WE, HILTON P, BIBBY J.: Tension-free vaginal tape for primary genuine stress incontinence: a two centre follow-up study. BJU International 2000; 86: 39-42.

49. ULMSTEN U, FALCONER C, JOHNSON P et al.: A multicenter study of tension-free vaginal tape (TVT) for surgical treatment of stress urinary incontinence. Int Urogynecol $J$ Pelvic Floor Dysfunct 1998; 9: 210-213.

50. ULMSTEN U, JOHNSON P, REZAPOUR M.: A three-year follow-up of tension-free vaginal tape for surgical treatment of female stress urinary incontinence. $\mathrm{Br} J$ Obstet Gynecol 1999; 106: 345-350.

51. JEFFRY L, DEVAL B, BIRSAN A, SORIANO D, DARAI E.: Objective and subjective cure rates after tension-free vaginal tape for treatment of urinary incontinence. Urology 2001; 58: 702-708.

52. NILSSON CG, KUUVA N, FALCONER C, REZAPOUR M, ULMSTEN U.: Long term results of the tension-free vaginal tape (TVT) procedure for surgical treatment of female stress urinary incontinence. Int Urogynecol $J$ Pelvic Floor Dysfunct 2001; 12: 5-8.

53. HAAB F, SANANES S, AMARENCO G et al.: Results of tension-free vaginal tape procedure for the treatment of type II stress urinary incontinence at a minimum follow up of 1 year. J Urol 2001; 165: 159-162.

54. KUUVA N, NILSSON CG.: A nation wide analysis of complications associated with the tension-free vaginal tape (TVT) procedure. Acta Obstet Gynecol Sacnd 2002; 81: 72-77.

55. CORCOS J, ABOUASSALY R, STEINBERG $J$ et al.: Complications of tension-free vaginal tape surgery: a multi-institutional review of 241 cases. $32^{\text {nd }}$ ICS Annual Meeting, august 28-30, 2002, Heildelberg (Alemania). Neurourol Urodynam 2002; 21: 4 (abstract 32).

56. PARK SC, HONG BS, SONG CR, KIM JB, CHOO MS.: Risk factors related to inmediate urinary retention after tension-free vaginal tape procedure. Eur Urol Supplements 2003; 2: 194 (Abstract 765).

57. WANG KH, WANG KH, NEIMARK M, DAVILA GW.: Voiding dysfunction following TVT procedure. Int Urogynecol $J$ Pelvic Floor Dysfunt 2002; 13: 353-358.

58. JACQUETIN B.: Use of TVT in surgery for female urinary incontinence. J Gynecol Obstet Biol Reprod 2000; 29: 242-247.

Dr. J.L. Gutiérrez Baños

C/ Francisco de Cáceres, 1L, 4ํㅜ centro

39012 Santander (Cantabria)

(Trabajo recibido el 1 julio de 2003) 